\title{
Étude sur la participation à la mammographie de dépistage et sur son utilisation au Canada
}

\author{
G. P. Doyle, M.B.A. (1); D. Major, Ph. D. (2); C. Chu, M. Sc. (3); A. Stankiewicz, M.H.P. (4); M. L. Harrison, M. Sc. (5); \\ L. Pogany, M. Sc. (4); V. M. Mai, M.D. (6); J. Onysko, M.A. (4)
}

Cet article a fait l'objet d'une évaluation par les pairs.

\section{Résumé}

Introduction : Le taux de participation est un indicateur important de l'efficacité d'un programme de dépistage; cependant, la méthode actuellement utilisée pour mesurer le taux de participation au Canada ne permet pas d'établir des comparaisons avec les autres pays. Cette étude vise à évaluer la méthode utilisée pour mesurer la participation à la mammographie de dépistage au Canada, à comparer les méthodes utilisées à l'échelle internationale et à proposer d'autres méthodes.

Méthodologie : Les données des programmes canadiens de dépistage du cancer du sein sur les femmes âgées de 50 à 69 ans ayant subi un examen de dépistage entre 2004 et 2006 ont été extraites de la Base de données canadienne sur le dépistage du cancer du sein (BDCDCS). Les données sur les mammographies effectuées par des médecins rémunérés à l'acte (mammographies de dépistage opportunistes) ont été fournies par les ministères provinciaux de la Santé. Les taux de participation aux programmes de mammographie de dépistage et les taux d'utilisation à des intervalles de 24 et de 30 mois ont été examinés.

Résultats : Au Canada, le taux de participation aux programmes de dépistage s'élève à 39,4\% selon un intervalle de 24 mois, et il est de 43,6\% lorsque l'intervalle est fixé à 30 mois. Toujours au Canada, le taux d'utilisation de la mammographie s'élève à 63,1\% selon un intervalle de 24 mois; il passe à 70,4 \% lorsque l'intervalle est de 30 mois.

Conclusion : Compte tenu des différences dans la prestation des services de santé d'une province canadienne à une autre, il faudrait surveiller tant la participation aux programmes que le taux d'utilisation global de la mammographie selon des intervalles de 24 et de 30 mois.

Mots-clés : sein, mammographie, détection précoce du cancer, cancer du sein, cancer, participation à un programme, utilisation des programmes, dépistage

\section{Introduction}

Le cancer du sein est la deuxième cause de mortalité par cancer chez les Canadiennes ${ }^{1}$. Selon les prévisions, environ 23200 femmes devraient apprendre qu'elles sont atteintes du cancer du sein en 2010, et 5300 devaient en décéder ${ }^{1}$. Le dépistage du cancer du sein est généralement perçu comme une intervention en santé profitable, en particulier chez les femmes âgées de 50 à 69 ans. Des essais cliniques randomisés et des méta-analyses laissent croire que les programmes de dépistage permettent de réduire le taux de mortalité de $25 \%$ à $30 \%{ }^{2-6}$; cependant, la déclaration systématique s'est avérée difficile. La réduction du taux de mortalité dans la population admissible au dépistage est directement liée au taux de participation 7 . Par conséquent, le fait d'utiliser la participation comme mesure provisoire offre aux programmes une méthode de déclaration systématique plus pratique.

Il n'existe aucune mesure normalisée de la participation à la mammographie de dépistage. Il est difficile de déterminer les taux de participation au dépistage à l'extérieur du cadre des études en raison des différents types de services de mammographie, qui peuvent être offerts soit dans le cadre de programmes organisés de dépistage du cancer du sein, soit par des établissements de soins de santé. Les programmes organisés de dépistage du cancer du sein ciblent et invitent les femmes admissibles, puis leur offrent un examen de dépistage (il s'agit généralement d'une mammographie bilatérale de dépistage à deux clichés effectuée tous les deux ans), un suivi en cas d'anomalie, puis un rappel après un examen de dépistage négatif ou ayant révélé une tumeur bénigne.

La façon de calculer la participation à l'échelle nationale et internationale varie considérablement, tout comme les intervalles de dépistage recommandés et les

Coordonnées des auteurs :

1. Programme de dépistage du cancer du sein de Terre-Neuve-et-Labrador, St. John's (Terre-Neuve-et-Labrador), Canada

2. Institut national de santé publique du Québec, Québec (Québec), Canada

3. BC Cancer Agency, Vancouver (Colombie-Britannique), Canada

4. Agence de la santé publique du Canada, Ottawa (Ontario), Canada

5. Cancer Care Manitoba, Winnipeg (Manitoba), Canada

6. Cancer Care Ontario, Toronto (Ontario), Canada

Correspondance : Gregory P. Doyle, Programme de dépistage du cancer du sein de Terre-Neuve-et-Labrador, 35, chemin Major, bureau 102, St. John's (Terre-Neuve-et-Labrador) A1A 4Z9; tél. : 709-777-5064; téléc. : 709-777-5069; courriel : gregory.doyle@easternhealth.ca 
taux de fidélisation, ce qui complique davantage la déclaration systématique. Malgré cela, la plupart des programmes déclarent systématiquement leur taux de participation; ils se sont donné pour cible un taux de participation de $70 \%$, cible fondée sur une estimation de la réduction de la mortalité ${ }^{\text {. }}$

En raison des différences marquées dans les méthodes de déclaration de participation à la mammographie de dépistage et de l'utilité de déterminer la méthode de déclaration la plus efficace, le Partenariat canadien contre le cancer a mis sur pied un groupe de travail mandaté pour 1) examiner les éléments justifiant l'objectif d'un taux de participation de $70 \%$ chez les femmes âgées de 50 à 69 ans et 2) proposer d'autres méthodes de calcul du taux d'utilisation de la mammographie systématique (tous les deux ans) qui refléteraient plus exactement l'usage que font les Canadiennes des mammographies de dépistage.

\section{Méthodologie}

Le groupe de travail a passé en revue une partie de la littérature sur les taux de participation aux programmes de dépistage du cancer du sein afin de recenser l'ensemble des définitions et des méthodes de calcul de la participation utilisées par les programmes de divers pays. Nous avons sélectionné deux définitions en ce qui concerne les méthodes de calcul : le taux de participation à un programme, c'est-à-dire la proportion de femmes âgées de 50 à 69 ans ayant subi un examen de dépistage dans le cadre d'un programme organisé de dépistage du cancer du sein au cours d'une période définie, parmi la population totale de femmes âgées de 50 à 69 ans (obtenue à partir des données de recensement); et le taux global d'utilisation, c'est-à-dire la proportion combinée de femmes âgées de 50 à 69 ans ayant passé une mammographie bilatérale (soit une mammographie de dépistage effectuée dans le cadre d'un programme organisé, soit une mammographie de dépistage effectuée hors du cadre d'un programme organisé, soit une mammographie diagnostique bilatérale effectuée hors du cadre d'un programme organisé dans une province qui n'a pas de code de facturation pour la mammographie de dépistage non effectuée dans le cadre d'un programme), parmi la population totale de femmes âgées de 50 à 69 ans (obtenue à partir des données de recensement).

Les données sur le dépistage effectué dans le cadre d'un programme ont été extraites de la Base de données canadienne sur le dépistage du cancer du sein (BDCDCS). Des détails sur chacun des programmes et sur les diverses méthodes de collecte de données pour la BDCDCS sont fournis ailleurs ${ }^{9}$. Les données sur les demandes de remboursement des médecins rémunérés à l'acte ayant effectué des mammographies (mammographies de dépistage opportunistes) ont été fournies par les ministères provinciaux de la Santé. Les calculs ont été effectués à l'aide des données couvrant la période de 2004 à 2006, qui sont les chiffres les plus récents tirés de la BDCDCS et des données sur les médecins rémunérés à l'acte. Lorsque cela était possible, des références croisées ont été établies entre les données des programmes organisés de dépistage et les données concernant les médecins rémunérés à l'acte pour éviter que les données ne figurent en double; seul un très petit nombre d'examens de dépistage en double ont été trouvés. L'analyse englobait des données provenant de sept provinces : la Colombie-Britannique, l'Alberta, la Saskatchewan, le Manitoba, l'Ontario, le Québec et Terre-Neuve-et-Labrador. Des données autodéclarées sur les mammographies de dépistage effectuées au cours des 24 mois précédant l'étude ont été tirées de l'Enquête sur la santé dans les collectivités canadiennes (ESCC) réalisée par Statistique Canada ${ }^{9}$.

Deux intervalles de dépistage, un de 24 mois et un de 30 mois, ont été utilisés à titre de périodes de référence pour évaluer le taux de participation aux programmes et

TABLEAU 1

Aperçu des lignes directrices des programmes de dépistage et des méthodes de calcul du taux de participation ${ }^{10-18}$

\begin{tabular}{|c|c|c|c|c|c|c|}
\hline Pays & Canada & Australie & Nouvelle-Zélande & Europe & Royaume-Uni & Hongrie \\
\hline Organisation & Provinciale & Nationale & Nationale & - & Nationale & Nationale \\
\hline $\begin{array}{l}\text { Méthode de } \\
\text { recrutement }\end{array}$ & $\begin{array}{l}\text { Participation } \\
\text { volontaire }\end{array}$ & $\begin{array}{l}\text { Participation } \\
\text { volontaire/invitation }\end{array}$ & $\begin{array}{l}\text { Participation } \\
\text { volontaire/invitation }\end{array}$ & Invitation & Invitation & Invitation \\
\hline Groupe d'âge ciblé & $50-69$ & $50-69$ & $50-69$ & $50-69$ & $50-70$ & $45-65$ \\
\hline $\begin{array}{l}\text { Numérateur du } \\
\text { taux de participation }\end{array}$ & $\begin{array}{l}\text { Nombre de } \\
\text { femmes soumises } \\
\text { au dépistage au } \\
\text { cours d'une } \\
\text { période de } 2 \text { ans }\end{array}$ & $\begin{array}{l}\text { Nombre de } \\
\text { femmes soumises } \\
\text { au dépistage } \\
\text { au cours d'une } \\
\text { période de } 2 \text { ans }\end{array}$ & $\begin{array}{l}\text { Nombre de } \\
\text { femmes soumises } \\
\text { au dépistage } \\
\text { au cours d'une } \\
\text { période de } 2 \text { ans }\end{array}$ & $\begin{array}{l}\text { Nombre de } \\
\text { femmes invitées } \\
\text { soumises au } \\
\text { dépistage }\end{array}$ & $\begin{array}{l}\text { Nombre de femmes } \\
\text { invitées soumises } \\
\text { au dépistage au } \\
\text { cours d'une période } \\
\text { de } 12 \text { mois }\end{array}$ & $\begin{array}{l}\text { Nombre de femmes } \\
\text { invitées soumises } \\
\text { au dépistage au } \\
\text { cours d'une période } \\
\text { de } 2 \text { ans }\end{array}$ \\
\hline $\begin{array}{l}\text { Dénominateur du } \\
\text { taux de participation }\end{array}$ & $\begin{array}{l}\text { Moyenne des } \\
\text { populations de la } \\
1^{\text {re }} \text { et de la } 2^{\mathrm{e}} \text { années } \\
\text { établie à partir du } \\
\text { recensement/de la } \\
\text { prévision }\end{array}$ & $\begin{array}{l}\text { Moyenne de la } \\
\text { population estimée } \\
\text { des } 2 \text { années } \\
\text { au } 30 \text { juin }\end{array}$ & $\begin{array}{l}\text { Estimations lissées } \\
\text { de la population } \\
\text { du recensement } \\
\text { sur } 2 \text { ans }\end{array}$ & Population invitée & $\begin{array}{l}\text { Population invitée } \\
\text { au cours d'une } \\
\text { période de } 12 \text { mois }\end{array}$ & $\begin{array}{l}\text { Population invitée } \\
\text { au cours d'une } \\
\text { période de } 2 \text { ans }\end{array}$ \\
\hline $\begin{array}{l}\text { Taux de } \\
\text { participation ciblé }\end{array}$ & $\geq 70 \%$ & $\geq 70 \%$ & $\begin{array}{l}\geq 70 \% \text { des femmes } \\
\text { âgées de } 45 \text { à } 69 \text { ans }\end{array}$ & $\begin{array}{l}\text { Acceptable }:>70 \% \\
\text { Souhaitable : }>75 \%\end{array}$ & $\begin{array}{l}\text { Minimum : } \geq 70 \% \\
\text { Ciblé }: \geq 80 \%\end{array}$ & $\begin{array}{l}\text { Acceptable : }>70 \% \\
\text { Souhaitable : }>75 \%\end{array}$ \\
\hline
\end{tabular}

Remarques : En plus d'accepter les participantes volontaires, certaines provinces canadiennes envoient également des lettres d'invitation aux membres de la population ciblée. Cette pratique diffère de celle utilisée en Australie et en Nouvelle-Zélande, où tous les programmes acceptent les participantes volontaires et envoient des lettres d'invitation, et de celle utilisée au Royaume-Uni et en Hongrie, où seules les femmes ayant reçu une lettre d'invitation sont acceptées. 
TABLEAU 2

Programmes canadiens de dépistage du cancer du sein : pratiques courantes en 2004 et en 2006

\begin{tabular}{lcccc}
\multicolumn{1}{c}{ Province } & $\begin{array}{c}\text { Date de début } \\
\text { du programme }\end{array}$ & $\begin{array}{c}\text { Groupe } \\
\text { d'âge ciblé }\end{array}$ & $\begin{array}{c}\text { Services mobiles de } \\
\text { dépistage offerts? }\end{array}$ & ECS offert? \\
\hline Colombie-Britannique & 1988 & $50-69$ ans & Oui & Non \\
Alberta & 1990 & $50-69$ ans & Oui & Non \\
Saskatchewan & 1990 & $50-69$ ans & Oui & Non \\
Manitoba & 1995 & $50-69$ ans & Oui & Non $^{\text {a }}$ \\
Ontario & 1990 & $50-69$ ans & Oui & Oui $^{\text {bi }}$ \\
Québec & 1998 & $50-69$ ans & Oui & Non \\
Terre-Neuve-et-Labrador & 1996 & $50-69$ ans & Non & Ouic $^{\text {con }}$ \\
\hline
\end{tabular}

Abréviations : ECS, examen clinique des seins.

a Service d'ECS offert par une infirmière ou une technologue jusqu'en octobre 2005.

b Service d'ECS offert par une infirmière dans $52 \%$ des emplacements.

c ECS effectué par une infirmière.

le taux global d'utilisation des services de dépistage du cancer du sein. L'intervalle de 24 mois représente une interprétation stricte des recommandations concernant l'intervalle de dépistage et les objectifs en matière de rendement, tandis que l'intervalle de 30 mois reflète de façon plus réaliste l'observance des recommandations concernant l'intervalle de dépistage.

\section{Résultats}

Des différences considérables ont été observées à l'échelle internationale dans l'organisation, les méthodes de dépistage et de recrutement ainsi que les groupes d'âge visés des divers programmes de dépistage du cancer du sein ${ }^{10-18}$; ces différences ont probablement une incidence sur la comparaison des taux de participation (tableau 1). Au Canada, les programmes organisés font état de la participation des femmes âgées de 50 à 69 ans en se basant sur un dénominateur correspondant à la moyenne des populations de première et de deuxième années établie à partir des estimations du recensement (tableau 2). En calculant la probabilité cumulative de subir une mammographie de dépistage subséquente dans le cadre d'un programme (taux de fidélisation) au Canada, par exemple, on a établi qu'environ $30 \%$ seulement de la population ciblée pour le dépistage subit une mammographie de dépistage dans l'intervalle visé de 24 mois (figure 1).

Au Canada, le taux de participation aux programmes de dépistage du cancer du sein était de 39,4 \% (intervalle de 24 mois) et de 43,6\% (intervalle de 30 mois). Un

\section{Analyse}

Il est essentiel de disposer d'une estimation précise de l'utilisation de la mammographie de dépistage si l'on veut évaluer l'impact du dépistage sur la mortalité. Les rapports sur les indicateurs de participation (dépistage dans le cadre d'un programme, utilisation et données autodéclarées) doivent tenir compte du contexte, des limites des méthodes de calcul et des intervalles de dépistage (24 mois contre 30 mois), ainsi que des pratiques propres aux programmes faisant l'objet de la comparaison.

Toutes les méthodes utilisées dans le contexte canadien comportent des limites. La pratique courante qui consiste à ne déclarer que les examens de dépistage effectués dans le cadre d'un programme exclut une quantité importante de mammographies de dépistage, ce qui entraîne une sousestimation substantielle de la réduction possible du taux de mortalité. Les estimations établies à partir des données autodéclarées sur l'utilisation de la mammographie sont uniformes d'une province à une autre; cependant, ces estimations sont basées sur les rappels envoyés aux participantes à l'enquête, ce qui, croit-on, entraîne une surestimation du comportement souhaité ${ }^{19}$. L'utilisation de la mammographie pourrait amplifier l'impact sur la mortalité étant donné que les mammographies effectuées par des médecins rémunérés à l'acte ne font pas appel à des méthodes telles que

\section{FIGURE 1}

Probabilité cumulative de subir une mammographie de dépistage ultérieure, par groupe d'âge, chez les femmes ayant subi une mammographie en 2000 ou en 2001

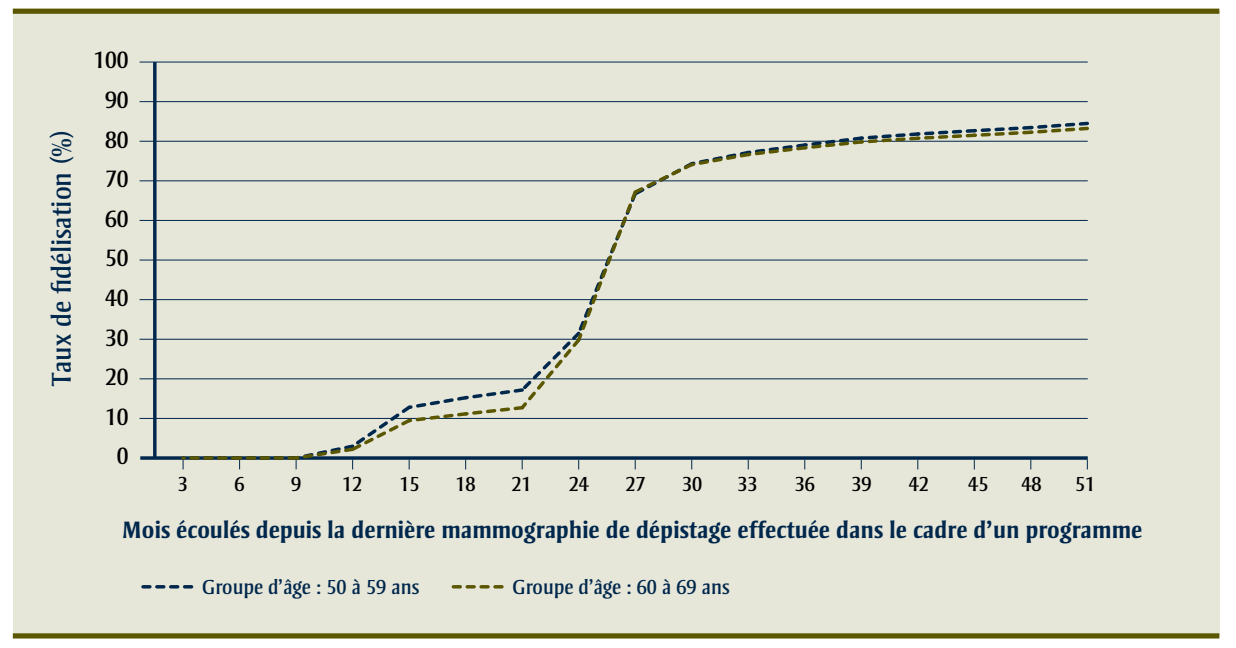


TABLEAU 3

Taux d'utilisation des programmes de dépistage et taux global d'utilisation, à l'échelle nationale et par province, 2004-2006

\begin{tabular}{|c|c|c|c|c|c|}
\hline Région & $\begin{array}{l}\text { Participation } \\
\text { au programme } \\
\text { sur } 24 \text { mois }^{\mathrm{a}} \\
\%\end{array}$ & $\begin{array}{c}\text { Participation } \\
\text { au programme } \\
\text { sur } 30 \text { mois }^{b} \\
\%\end{array}$ & $\begin{array}{l}\text { Taux global } \\
\text { d'utilisation } \\
\text { sur } 24 \text { mois }^{\text {a }} \\
\%\end{array}$ & $\begin{array}{l}\text { Taux global } \\
\text { d'utilisation } \\
\text { sur } 30 \text { mois } \\
\text { \% }\end{array}$ & $\begin{array}{l}\text { Femmes ayant déclaré avoir } \\
\text { subi une mammographie } \\
\text { de dépistage dans les } 24 \text { mois } \\
\% \text { (IC à } 95 \%)\end{array}$ \\
\hline$\%$ & 39,4 & 43,6 & 63,1 & 70,4 & $62,5(60,9-64,1)$ \\
\hline Colombie-Britannique $^{\mathrm{d}}$ & 51,1 & 55,1 & 60,0 & 65,4 & $60,1(55,7-64,6)$ \\
\hline Albertad $^{d}$ & 9,1 & 10,8 & 62,8 & 70,9 & $64,0(58,4-69,3)$ \\
\hline Saskatchewan $^{\mathrm{d}}$ & 48,3 & 54,8 & 60,9 & 68,8 & $63,7(58,1-69,2)$ \\
\hline Manitoba ${ }^{\mathrm{d}, \mathrm{e}}$ & 52,5 & 56,5 & 63,7 & 69,4 & $56,1(50,1-62,1)$ \\
\hline Ontario $^{d}$ & 32,4 & 36,5 & 63,5 & 72,5 & $62,7(59,8-65,7)$ \\
\hline Québec $^{\mathrm{d}}$ & 51,6 & 56,7 & 64,6 & 70,4 & $64,3(61,0-67,6)$ \\
\hline Terre-Neuve-et-Labrador ${ }^{d}$ & 35,4 & 36,6 & 63,9 & 68,6 & $61,5(55,0-68,1)$ \\
\hline
\end{tabular}

Abréviations : IC, intervalle de confiance.

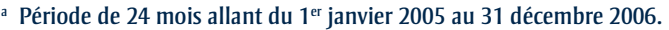

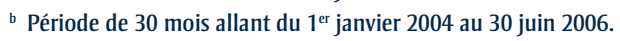

c Enquête sur la santé dans les collectivités canadiennes de $2008^{9}$

d Définitions des codes provinciaux pour la rémunération à l'acte disponibles sur demande.

e Période de 24 mois allant du 1 ${ }^{\text {er }}$ avril 2005 au 31 mars 2007; période de 30 mois allant du 1 1 avril 2004 au 30 septembre 2006.

le recrutement en population générale, les rappels automatiques pour des dépistages ultérieurs, un suivi coordonné des résultats anormaux à la suite d'un dépistage, une assurance systématique de la qualité ou l'évaluation régulière du rendement des programmes. De plus, le fait de classer par erreur des mammographies diagnostiques comme étant des mammographies de dépistage et de compter en double certains examens de dépistage subis chez un médecin rémunéré à l'acte ou dans le cadre d'un programme peut gonfler artificiellement le taux d'utilisation et mener à une surestimation de la réduction possible du taux de mortalité. En outre, les programmes n'étaient pas tous en mesure d'éviter la double comptabilisation de données lors du calcul du taux d'utilisation, mais ceux qui en étaient capables ont trouvé une quantité négligeable de données comptées en double. Les données autodéclarées sur l'utilisation et le taux global d'utilisation selon un intervalle de 24 mois sont semblables (tableau 3), ce qui laisse croire que les deux méthodes pourraient être plus précises que ne l'indiquaient des rapports antérieurs, ou du moins qu'elles comportent un biais semblable.

L'utilisation d'un intervalle de dépistage de 30 mois pour tenir compte de l'intervalle de dépistage " réel » entraîne une surestimation du nombre de mammographies de dépistage subies aux deux ans (figure 1). Bien que les résultats du dépistage, y compris le taux de rappel en raison d'une anomalie et le taux de détection d'un cancer, semblent très peu varier selon que les femmes subissent un nouveau dépistage après 30 mois ou après 24 mois $^{9}$, le fait de retarder le diagnostic et le traitement de seulement 3 à 6 mois pourrait être associé à une diminution du taux de sur$v_{i e}{ }^{20}$. Toutefois, en comparant les résultats obtenus chez les femmes ayant subi un dépistage annuel à ceux obtenus chez les femmes ayant subi un dépistage aux trois ans, les auteurs d'une étude sur la fréquence du dépistage du cancer du sein au Royaume-Uni ont constaté, au moyen d'indicateurs indirects de résultats, que la fréquence du dépistage avait un effet relativement faible sur le taux de mortalité attribuable au cancer du $\operatorname{sein}^{21}$. Compte tenu de ces données contradictoires, il est difficile de déterminer si les programmes de dépistage qui obtiennent un taux de participation de $70 \%$ sur un intervalle de 24 mois donneront de meilleurs résultats en termes d'incidence sur le taux de mortalité que les programmes qui obtiennent le même taux de participation sur un intervalle de dépistage de 30 mois.

Il convient de souligner le faible taux de participation à un programme de dépistage en Alberta comparativement au taux observé pour l'ensemble du Canada $(9,1 \%$ contre 39,4 \%) (tableau 3). Durant la période visée par l'étude, c'est-à-dire de 2004 à 2006, le programme organisé de dépistage du cancer du sein en l'Alberta offrait des services dans deux centres fixes situés dans deux des principales villes de la province ainsi qu'un service mobile dans les régions éloignées. Ailleurs dans la province, des services de mammographie étaient également accessibles par l'intermédiaire du système de rémunération à l'acte. Ainsi, dans l'ensemble, la province a obtenu des taux d'utilisation de la mammographie comparables aux taux nationaux (70,9 \% contre $70,4 \%$ respectivement). Un programme de dépistage du cancer du sein a été lancé à la grandeur de la province en mars 2008.

Il est extrêmement difficile de faire en sorte que les taux de participation soient comparables à l'échelle internationale. Un indicateur doit avoir le plus petit biais possible et refléter de manière exacte les pratiques qui ont la plus grande incidence sur la réduction du taux de mortalité. En outre, au moment de faire des comparaisons, il faut toujours prendre en considération les pratiques propres aux divers programmes. Alors que la plupart des programmes fournissent des données sur les femmes âgées de 50 à 69 ans, les programmes offerts en Hongrie et au Royaume-Uni utilisent un groupe d'âge plus large (tableau 1). La plupart des programmes envoient un rappel aux deux ans, mais le Royaume-Uni le fait aux trois ans. Plus important encore, les méthodes de calcul du dénominateur et du numérateur varient considérablement, la méthode utilisée au Canada étant 
celle qui permet d'obtenir l'estimation la plus conservatrice du taux de participation (tableau 1).

\section{Conclusion}

En général, les mesures du taux global d'utilisation de la mammographie de dépistage au Canada laissent croire que les objectifs fixés sont presque atteints, mais il est impossible de présumer que l'utilisation globale a le même impact sur la mortalité que la participation aux programmes de dépistage. Cela est attribuable à la quantité insuffisante de données sur la qualité des mammographies de dépistage effectuées par des médecins rémunérés à l'acte.

En raison des différences observées entre les provinces quant aux structures de soins de santé et la prestation des services, et vu le nombre important de mammographies opportunistes qui sont effectuées au Canada, nous concluons qu'il faudrait surveiller tant la participation aux programmes que le taux global d'utilisation de la mammographie à des intervalles de 24 et de 30 mois, et faire rapport à ce sujet. Nous sommes également d'avis que la production de rapports sur plusieurs indicateurs de participation pourrait faciliter la comparaison des données sur l'utilisation de la mammographie à l'échelle internationale.

\section{Remerciements}

Cette recherche a été financée par le Partenariat canadien contre le cancer. Les auteurs tiennent à remercier Laura McDougall des Services de santé de l'Alberta pour les explications et les précieux renseignements qu'elle a fournis.

\section{Références}

1. Comité directeur de la Société canadienne du cancer du sein. Statistiques canadiennes sur le cancer 2010. Société canadienne du cancer. Toronto (Ont.) : Société canadienne du cancer; 2010.
2. Andersson I, Aspegren K, Janzon L, Landberg $\mathrm{T}$, Lindholm $\mathrm{K}$, Linell $\mathrm{F}$, et al. Mammographic screening and mortality from breast cancer: the Malmo mammographic screening trial. BMJ. 1988;297:943-8.

3. Alexander FE, Anderson TJ, Brown HK, Forrest AP, Hepburn W, Kirkpatrick AE, et al. 14 years of follow-up from the Edinburgh randomised trial of breast-cancer screening. Lancet. 1999;353;1903-08.

4. Duffy SW, Tabar L, Vitak B, Yen MF, Warwick J, Smith RA, et al. The Swedish Two-County Trial of mammographic screening: cluster randomisation and end point evaluation. Ann Oncol. 2003;14(8):1196-8.

5. Shapiro S, Strax P, Venet L. Periodic breast cancer screening in reducing mortality from breast cancer. JAMA. 1971;215:1777-85.

6. International Agency for Research on Cancer Screening. IARC Handbooks of Cancer Prevention, Vol.7, Breast Cancer Screening. Lyon (FR) : IARC Press; 2002.

7. Day NE, Williams DR, Khaw KT. Breast cancer screening programmes: the development of a monitoring and evaluation system. Br J Cancer. 1989;59(6):954-8.

8. Forrest AP. Breast cancer: the decision to screen. 4th H.M. Queen Elizabeth The Queen Mother Fellowship; 1990. London (UK): Nuffield Provincial Hospitals Trust; 1990.

9. Agence de la santé publique du Canada. Programmes organisés de dépistage du cancer du sein au Canada : rapport sur le rendement des programmes en 2005 et en 2006. Ottawa (Ont.) : Santé Canada. À paraître en 2011.

10. Groupe de travail sur les indicateurs d'évaluation. Lignes directrices pour la surveillance de la performance des programmes de dépistage du cancer du sein, 2e éd. Ottawa (Ont.) : Agence de la santé publique du Canada; mars 2007.
11. BreastScreen Australia Data Dictionary Version 1 [Internet]. [lieu inconnu]: National Quality Management Committee; 2004 [consultation le 7 mars 2009]. PDF (887 Ko) téléchargeable à partir du lien : http://www.cancerscreening.gov.au/internet /screening/publishing.nsf/Content/br -dictionary/\$File/bsa-dd.pdf

12. BreastScreen Aotearoa Data Management Manual Version 4.0 [Internet]. [lieu inconnu]: National Screening Unit BreastScreen Aotearoa; 2010 Mar. PDF (1,29 Mo) téléchargeable à partir du lien : http://www.nsu.govt.nz/files/BSA/Data_ management_manual.pdf

13. Consolidated guidance on standards for the NHS Breast Screening Programme. NHSBSP Publication No 60 version 2. London (UK): NHS Cancer Screening Programmes; 2005 Apr.

14. Boncz I, Sebestyen A, Dobrossy L, Pentek Z, Budai A, Kovacs A, et al. The organisation and results of first screening round of the Hungarian nationwide organized cancer screening programme. Ann Oncol. 2007;18:795-9.

15. National Quality Management Committee of BreastScreen Australia. BreastScreen Australia National Accreditation Standards, Quality Improvement Program. BreastScreen Australia; juillet 2001, revu en avril 2008.

16. Breastscreen Aotearoa [Internet]. New Zealand National Health Board; 2009 [consultation le 7 mars 2009]. Consultable en ligne à la page : http://www.nsu.govt.nz /Current-NSU-Programmes/559.asp

17. Taylor R, Arnett K, Begg S. BreastScreen Aotearoa Independent Monitoring Report, January-June, 2007 [Internet]. Queensland (AU): School of Population Health, University of Queensland; 2007 [consulté le 7 mars 2009]. Consultable en ligne à la page : http://www.nsu.govt.nz/Health -Professionals/1048.asp 
18. Perry N, Broeders M, De Wolf C, Tornberg S, Holland R, Von Karsa L, Puthaar E, éditeurs. Lignes directrices européennes concernant l'assurance de qualité dans le dépistage du cancer du sein et le diagnostic, 4e éd. Direction générale Santé et protection des consommateurs, Communautés européennes : Bruxelles (LU); 2006.

19. Bancej CM, Maxwell CJ, Snider J. Inconsistent self-reported mammography history: findings from the National Population Health Survey longitudinal cohort. BMC Health Serv Res. 2004;4:32.

20. Richards MA, Westcombe AM, Love SB, Littlejohns P, Ramirez AJ. Influence of delay on survival in patients with breast cancer: a systematic review. Lancet. 1999;353(9159):1119-26.

21. Breast Screening Frequency Trial Group. The frequency of breast cancer screening: results from the UKCCCR Randomised Trial. United Kingdom Co-ordinating Committee on Cancer Research. Eur J Cancer. 2002;38:1458-64. 